\title{
Research on coalescence and probabilistic failure physics modeling of coplanar semiellipse cracks
}

\author{
Sufen Li, Yufeng Sun ${ }^{\mathrm{a}}$, Weiwei Hu and Zhaoxia Liu \\ Reliability and System Engineering School, Beihang University, China
}

\begin{abstract}
Multiple fatigue cracks, causing transient fatigue fracture and resulting in great loss of life and property, are very commen in engineering structures. In order to predict the fatigue life of engineering alloys more accurately, the interaction and coalescence of adjacent cracks was investigated and the four stages of cracks growth were discussed in this paper. The SIF(stress intensity factor) value was found to growth rapidly in the ligament failure, and simulation method was used to find out the coalescence point. Then, the Paris' law was chosen to discribe the crack growth process. Taking random factors like structure size, material properties, environment stress parameter into account, the probability physical of failure model of alloys can predict the failure life more accurate. Accordingly, the uncertain parameters of the Paris' law were updated via Bayesian update method. The posterior distributions of uncertain parameters were obtained by the MCMC-Gibbs sampling. Finally, the probabilistic failure life models before and after coalescence were established by Monte Carlo simulation method. The probabilistic modeling method, which can greatly reduces the historical data or experimental data needed for the reliability evaluation of small sample products, can also be applied to other aspect of physic of failure, effectively saving time and cost.
\end{abstract}

\section{Introducition}

Fatigue failures, which occures at stress below the tensile strength or yield strength of alloys is hard to be detected. Since most engineering components are or similarly are subjected to alternating stress. Multiple fatigue cracks causing transient fatigue fracture and resulting in great loss of life and property, are very commen in engineering structures. Crack mainly initiats in localized areas of stress concentratin or material defects[1,2]. Then these initial cracks grow together by interacting with each other, meanwhile shortening the fatigue life with an accelerated crack growth rate[3]. Interaction and coalescence processes of adjacent cracks is the key issue in the study of coplanar fatigue cracks.

Various theories have been propounded to assess the growth of adjacent surface cracks growth. The British Standard Institution[4] proposed a prediction method assuming cracks to be indepentend. However this idealize simple model mainly considering the geometric condition of cracks, turned out to be too conservative[5]. Iida[6] assumed that when the cracks touch, a semi-elliptical crack immediately drawn through the outer tips of the two adjucent cracks and through the deepest point of the deeper crack. The study neglected the interaction in calculation between adjucent cracks(the interaction factors $\gamma \mathrm{A}$ to $\mathrm{F}=1$ ) and proposed a simple realistic prediction method. However the

\footnotetext{
${ }^{\text {a }}$ Corresponding author : syf@buaa.edu.cn
}

(C) The Authors, published by EDP Sciences. This is an open access article distributed under the terms of the Creative Commons Attribution License 4.0 (http://creativecommons.org/licenses/by/4.0/). 
SIF(stress intensity factor) approxination and the material constents used may result in unsafe assessments. Leek \& Howard [7, 8] developed methods to calculates the interaction factors $\gamma \mathrm{A}$ to F, which quantifies the interactions between the stress intensity factors of adjucent cracks. But the coalescence point have not figured out.

The Physics of failure $(\mathrm{PoF})$ model can characterize the relation of the time of failure, the degradation of the performance parameters, the structure, the material and so on. The PoF model can describe the failure cause from the failure mechanism level and predict the failure time. [9-11]. After twenty-first Century, researchers gradually realized that the deterministic model could not describe the inevitability of material, stress and other conditions. Thus the PoF model tend to develope into the Probabilistic Physics of failure( $\mathrm{PPoF}$ ), which describes the probability function relation between the external environment and the time of failure. [12-14]

In this paper, 1) the coalescence of two adjucent coplanlar semiellipse cracks is researched via simulation method; 2) probabilistic failure physics $(\mathrm{PPoF})$ models for cracks before and after coalescence are established; 3) life distribution of probability density curves are obtaind by Monte Carlo simulation.

\section{Methods}

\subsection{Crack interaction and coalescence process}

According to Bayley[1], fatigue crack coalescence means two adjacent crack increase in size and finally grow together to form a single lager crack. The adjacent and coplanar surface cracks under LEFM conditions can interact. And Leek[7]et al. estimated the interaction factors for two coplanar surface cracks. The interaction and coalescence between adjucent cracks can critically increase the crack growth rate(Fig. 1). To assess the fatigue life of engineering components safely, crack interaction and coalescence must be taken into consideration. As illustrated in Fig. 2, the interaction and coalescence process can be described in four stages:

1) Independent stage: the spacing of crack tips are large enough that the two adjacent cracks can be regarded as independent.

2) Interaction stage: the two crack get closer and start to influences the stress field of each other.

3) Recharacterization stage: the neiboring cracks are close enough to form a concave crack. The front of the concave crack growth rapidly due to the high SIF value, which is called the failure of ligament. The stage ends when the concave crack is recharacterized as an envelope crack.

4) Post-coalescence stage: the envelope crack tends to resume its semi-cicular shape. Afterwards, it continues to popagate as it did before the interaction.

It's not easy to define the recharacterization stage, for it's hard to be observed. Moukawsher et al.[16] demonstrated that it is the yeild failure of the ligament that caused the failure of small fatigue cracks. And Swift[17] applied a yeild criterion to a linear alastic solution of the crack tip stress field. In the studies of Jeong[18], it was assumed that the yielded volumes was resulted from the stress field at each crack tip. Owing to the constraint of the surrounding material preventing complete plastic deformation, the stress concentrated at the center of the ligament. Once the average ligament stress reach a critical value that is equivalent to the ultimate tensile strength, the ligament failure occurs.
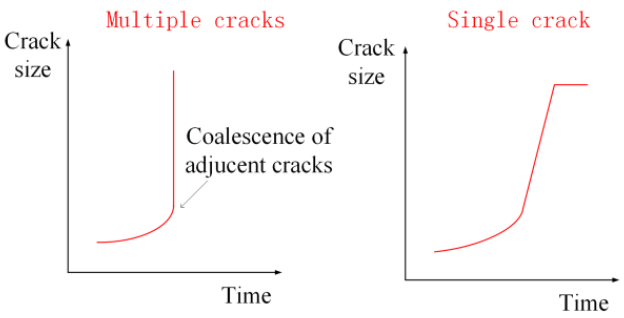

Figure 1. The crack growth rate of multiple cracks and single cracks. 


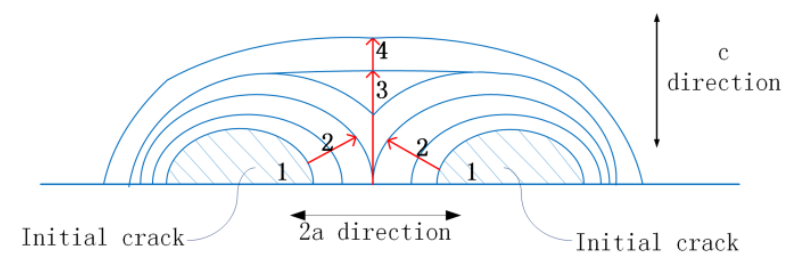

Figure 2. The interaction and coalescence process of adjucent cracks.

\subsection{The line spring model of multi coplanar cracks}

To study the interaction and coalescence of multiple co-plane cracks, the numerical simulation method is used to calculate the stress intensity factor. Compared with the crack size, the inelastic region of the crack tip is very small, so linear elastic fracture mechanics (LEFM) can be applied to solve the fatigue problem. Rice proposed that the issue of three dimensional surface crack can be simplified to the plate and shell structure with line spring model.The line spring model is a simple and effective method to solve the problem of co-plane multiple cracks[3, 14, 15].

In the line spring model, there is a infinite plate with n coplanar surface cracks under force $N^{\infty}$ and bending moment $M^{\infty}$ (Fig. 3(a)) ai and ci refers to the depth and half length of the crack i; bi and di means the coordinates of the crack tips on the front surface of crack $i$, where $i=1,2,3, \ldots \ldots$. As shown in Fig. 3(b) the model is simplified to a plate containing $\mathrm{n}$ semi - elliptical cracks, the discontinuities of cracks are treated with line springs.. When $\mathrm{x} \in\left[b_{i}, d_{i}\right]$, the line springs can be discribed with a plane strain slab. The SIF of the surface crack front is equal to the SIF of the corresponding position of the edge crack on the slab.

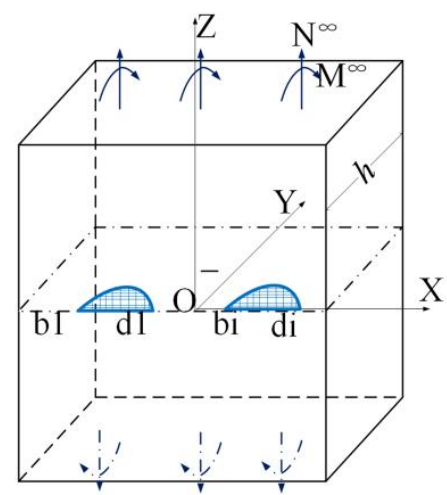

(a)

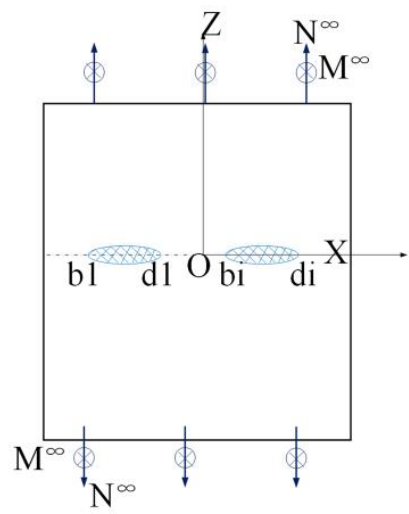

(b)

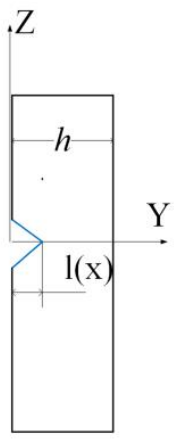

(c)

Figure 3. The line spring model

Accroding to Sun[3], the dimensionless integral characteristic equations of the plate with $n$ cracks are:

$$
\left\{\begin{array}{c}
\sum_{j=1}^{n} \int_{-1}^{1} \frac{1}{t_{j}-x_{i}}\left(\frac{c_{j}}{c_{1}}\right) \mu_{j 1}(\bar{t}) d \bar{t}=\frac{2 \pi}{E}\left[\sigma_{i M}\left(\overline{x_{l}}\right)-\sigma_{M}{ }^{\infty}\right] \\
\sum_{j=1}^{n} \int_{-1}^{1}\left[\frac{1}{t_{j}-x_{i}}+k\left(t_{j}, x\right)\right]\left(\frac{c_{j}}{c_{1}}\right) \mu_{j 2}(\bar{t}) d \bar{t}=\frac{4 \pi}{E}\left(\frac{c_{1}}{h}\right)\left[\sigma_{i B}\left(\bar{x}_{l}\right)-\sigma_{B}{ }^{\infty}\right]
\end{array}\right.
$$

where $t_{j}=\frac{c_{i}}{c_{1}} \bar{t}+\frac{b_{i+d_{i}}}{2 c_{1}}, \quad x_{j}=\frac{c_{i}}{c_{1}} \bar{x}+\frac{b_{i+d_{i}}}{2 c_{1}} ; \bar{t}, \bar{x}[-1,1] ; \mathrm{E}$ refers to the modulus of elasticity; $k\left(t_{j}, x\right)$ is known kernel function; ci refers to half length of the crack; $h$ is the slab thickness; $\sigma_{M}{ }^{\infty}=N^{\infty} / h, \sigma_{B}^{\infty}=6 M^{\infty} / h^{2} ; \mu_{j 1}(\bar{t}), \mu_{j 2}(\bar{t})$ are unknown dislocation density function; $\sigma_{i M}\left(\bar{x}_{l}\right)$, $\sigma_{i B}\left(\bar{x}_{l}\right)$ are unknown stesses; $\mathrm{i}=1,2,3, \ldots, \mathrm{n}$. 
The line spring constitutive relation is determined by the relationship between the generalized force suffered by the plane strain edge slab and the additional generalized displacements caused by cracks. Taking crack i for example:

$$
\left\{\begin{array}{c}
\sigma_{i M}\left(\bar{x}_{i}\right) \\
\sigma_{i B}\left(\bar{x}_{i}\right)
\end{array}\right\}=\frac{E}{1-v^{2}}\left[\begin{array}{ll}
\gamma_{M M}^{(i)} & \gamma_{M B}^{(i)} \\
\gamma_{B M}^{(i)} & \gamma_{B B}^{(i)}
\end{array}\right]\left\{\begin{array}{c}
\frac{c_{i}}{h} \int_{-1}^{1} \mu_{i 1}(\bar{t}) H(\bar{x}-\bar{t}) d \bar{t} \\
\frac{1 c_{i}}{6 c_{1}} \int_{-1}^{1} \mu_{i 2}(\bar{t}) H(\bar{x}-\bar{t}) d \bar{t}
\end{array}\right\}
$$

where the matrix $\left[\gamma_{\lambda \mu}^{(i)}\right]=\left[\alpha_{\lambda \mu}^{(i)}\right]^{-1}, \quad \mathrm{H}(\bar{x}-\bar{t})$ are unit step functions; $\alpha_{\lambda \mu}^{(i)}=\int_{0}^{\xi} g_{\lambda}^{(i)}(\xi) g_{\mu}^{(i)}(\xi) d \xi(\lambda, \mu=M, B) ; g_{M}^{(i)}(\xi)$ and $g_{B}^{(i)}(\xi)$ are known function.

Under the general force $\mathrm{N}(\mathrm{x})$ and $\mathrm{M}(\mathrm{x})$, the stress intensity factor of the edge crack slab can be described as :

$$
\mathrm{K}_{I}=\sqrt{h}\left[\sigma_{M}(\overline{\mathrm{x}}) \mathrm{g}_{M}(\xi)+\sigma_{B}(\bar{x}) \mathrm{g}_{B}(\xi)\right]
$$

where $\xi=1(x) / h$, and $1(x)$ means the distance between the tip of the edge crack and the axis of the ellipse(Fig. 3(c)). Substitut equation (2) into equation (3), the basic integral equations for the surface crack line spring model with residual stress are acquired and it can be solved by the Gauss-Chebyshev methods[2]. Afterwards, the stress intensity factor near the crack tip can be solved by using equation (1) and equation (3).

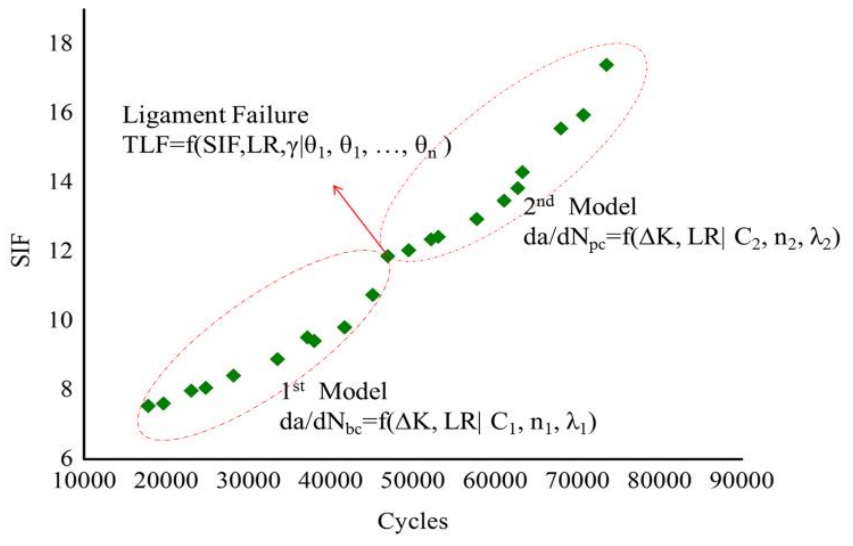

Figure 4. The simulation results of SIF of coplanar cracks.

Simulations have been done to observe the change of the SIF of coplanar cracks under the stress $290 \mathrm{Mpa}$. As can be seen from Fig. 4, the SIF value increases with the fatigue cycling number. And there is a sudden increase of the SIF near where fligament failure is expect to occur. Cracks growth with different crack propagation speed before and after the fligament failure. Thus, there are two failure physics model to describe the fatigue life.

\subsection{Physic of failure model}

\subsubsection{The Paris law}

The opening mode caused by tensile stress, the most common cracks in engineering, will be studied in this paper. Based on the experiment, the Paris's law was promoted by Paris and Erdogan in 1963[22]. Taking the dislocation dynamics theory, the thermal activation energy theory and the rate process into account, sevral investigator revealed the physical essence of the Paris equation[23, 24]:

$$
\frac{d a}{d N}=\frac{A_{0}}{f^{\lambda}}(\Delta K)^{\lambda} \exp \left(-\frac{\lambda U}{k T}+\frac{\alpha \lambda l n \Delta K}{k T}\right)
$$


$d a / d N$ means the crack growth rate and $\mathrm{f}$ is the lading frequency; $\Delta \mathrm{K}$ is the stress intensity factor. And

$$
A_{0}=b \gamma(m)\left(\frac{A}{4 b G \epsilon^{1 / 2}}\right)^{\lambda}
$$

where $\mathrm{b}$ is the Burgers vector; $\gamma(\mathrm{m})$ is a dimensionless quantity about $\mathrm{m} ; \mathrm{G}, \tau_{a}$ means shear modulus and shear stress respectively; $\varepsilon$ is the placement under mean stress; $\lambda=(m+1) /(m+2)$;

Denote as:

$$
\begin{gathered}
\mathrm{C}=\frac{A_{0}}{f^{\lambda}} \exp \left(-\frac{\lambda U}{k T}\right) \\
\mathrm{n}=\lambda+\frac{\alpha \lambda}{k T}
\end{gathered}
$$

Thus, the famous Paris equation is abtained:

$$
\frac{d a}{d N}=C(\Delta K)^{n}
$$

To take the loading ratio (lr) into consideration, a parameter $m$ is introduced in this paper:

$$
\frac{d a}{d N}=C(\Delta K)^{n}(l r)^{m}
$$

\subsubsection{Model parameters}

In the Paris equation, there are two uncertain parameter: $C, n$ and $m$, whose prior data are collected by researching literatures.

The prior information matrix are as following:

$$
\theta_{C, n}=\left[\begin{array}{cc}
C & n \\
2.06 E-11 & 4.59 \\
6.11 E-14 & 4.42 \\
5.13 E-10 & 3.61 \\
3.00 E-10 & 2 \\
4.53 E-10 & 2.09 \\
1.13 E-10 & 2
\end{array}\right]
$$

After fitting these data, the prior distribution of the parameter $\mathrm{C}$ and $\mathrm{n}$ are obtained: $\mathrm{C}_{\mathrm{B}} \sim \operatorname{Normal}(2.33 \mathrm{E}-10,2.41 \mathrm{E}-10), \mathrm{n}_{\mathrm{B}} \sim \operatorname{Normal}(3.10,1.19)$. Besides, the uncertain parameter $m$ is assumed as uniform distribution: $\mathrm{m} \sim \operatorname{Uniform}(-4,4)$.

\subsection{Probabilistic failure physics}

The PoF model can establish the relationship between failure time or the degradation of performance parameters with structure, material, work environmental conditions and function, describing the failure cause from the failure mechanism level and predicting the product failure time. However, owing to the uncertainties of structure size, material properties, environment stress parameter, the product life is supposed to follow a certain distribution. Based on the PoF model and taking these random factors into account, the probability failure of physical model can describ the failure life more detail.

Fig. 5, shows the realization way for PPoF. Firstly, analysis the failure mechanism of product according to its working environment and stress conditions, and choose a appropriate PoF model. Secondly, analysis and research on the uncertainties of the failure mechanism, and get the prior distribution of the uncertain parameters by fitting the investigation data. Furthermore, collect evidence data by simulation experments and obtain the likelihood function of crack growth rate. Then, update 
the parameters according to the Bayeslaw to get the posterior distribution; due to the difficulty of high dimensional integral calculation process, the MCMC-Gibbs sampling method is used to aquire the posterior distribution of the parameters. Finally, the posterior distribution of the failure life is acquired through Monte Carlo sampling.

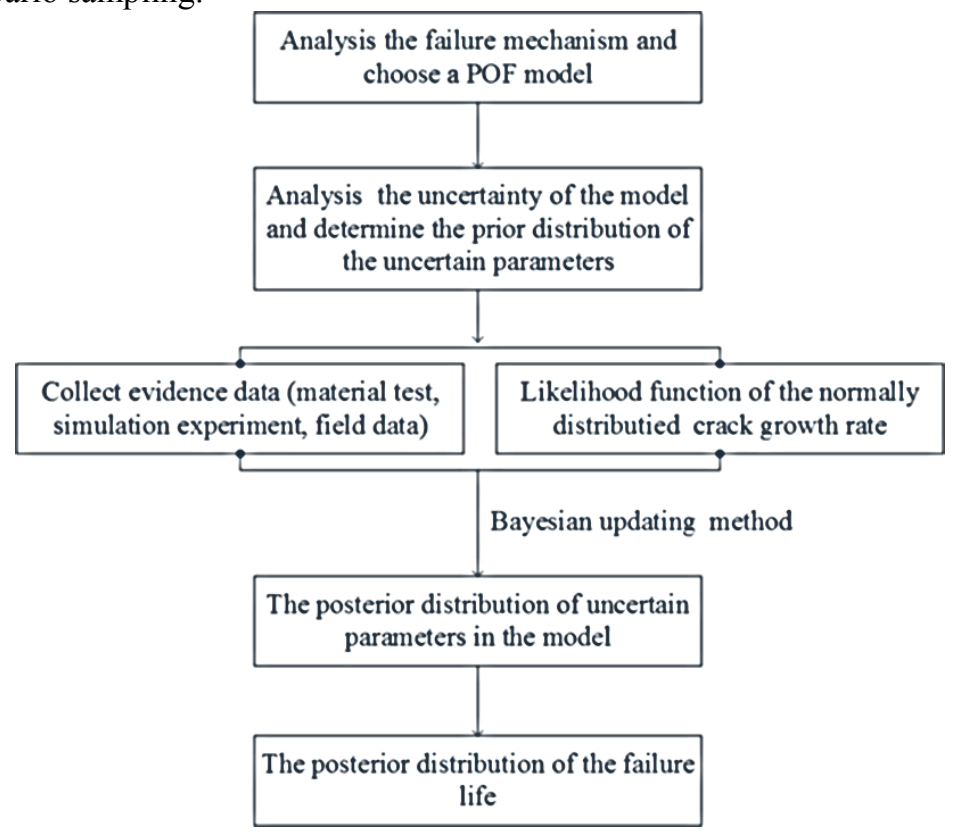

Figure 5. The flow-process diagram of realization way for PPoF.

\section{Results}

The evidence data (Ds) required in the Bayes updating comes from the simulation experiments. Assuming the likehood function of the crack growth rate that the data obey normal distribution:

$$
L(C, l, m, \sigma \mid D s)=\prod_{i=1}^{T} \frac{1}{\sigma \sqrt{2 \pi}} e^{\frac{\frac{d a}{d N i}-\left(C \Delta K^{n}(l r)^{m}\right)}{2 \sigma^{2}}}
$$

According to the Bayes equation, the posterior distribution of the parameters are as following :

$$
\pi(C, m, n, \sigma \mid D s)=\frac{L(C, m, n \mid D s) \pi_{0}(C, m, n)}{\iiint L((C, m, n \mid D s)) \pi_{0}(C, m, n) d C d m d n}
$$

Obviously, it is difficult to solve the above equation analytical with integral method. However, with the usage of WinBUGS, it can be solved by using Markoff Montecalo method. Combination the prior information and test data with crack propagation, the Bayesian parameter updating method can obtain the probabilistic failure physics model. To study the change of the parameters, model parameters before and after the failure of the ligament are updated respectively.

\subsection{The model before coalescence}

Fig. 6 presents the posteir distribution of uncertain parameters before coalescence, which are required in the Bayesian parameter updating. The mean value of uncertain parameters in Table 1. are used to establish the failure life model. And the mean value of $\mathrm{C}, \mathrm{m}, \mathrm{n}$ are 2.376E-10, 0.1997, 2.09, respectively. Thus the mean model for cracks before coalescence is formula (12). 

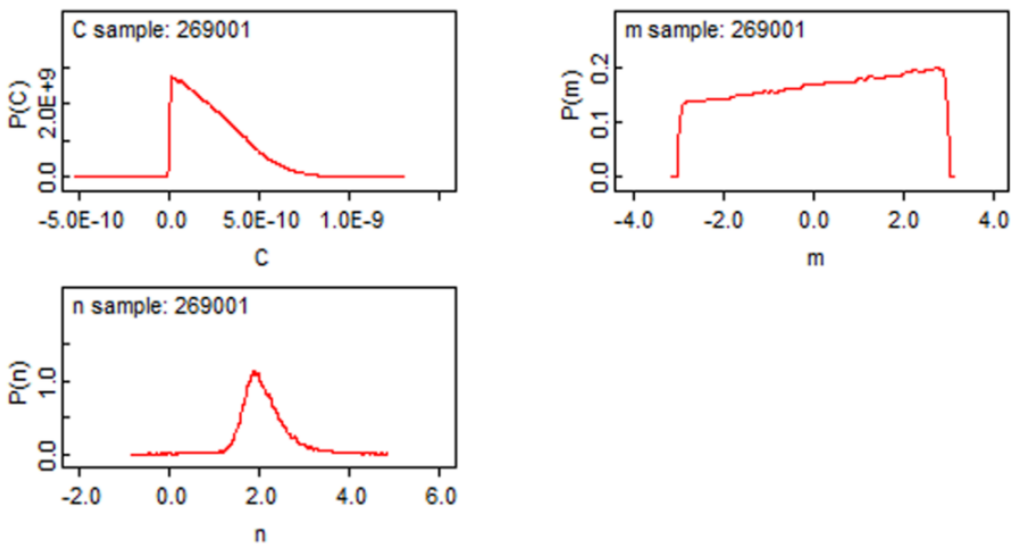

Figure 6. The posteir distribution of uncertain parameters before coalescence

Table 1. The updating results of uncertain parameters before coalescence

\begin{tabular}{|l|l|l|l|l|l|l|}
\hline & Mean & sd & MC_error & Val2.5pc & median & Val97.5pc \\
\hline$C$ & $2.376 \mathrm{E}-10$ & $2.027 \mathrm{E}-10$ & $2.986 \mathrm{E}-12$ & $8.788 \mathrm{E}-10$ & $2.047 \mathrm{E}-10$ & $6.474 \mathrm{E}-10$ \\
\hline$m$ & 0.1997 & 1.722 & 0.009009 & -2.813 & 0.292 & 2.876 \\
\hline$n$ & 2.09 & 0.46 & 0.01052 & 1.393 & 2.019 & 3.216 \\
\hline
\end{tabular}

$$
\frac{d a}{d N}=2.376 E-10 \Delta K^{0.197}(l r)^{2.09}
$$

\subsection{The model after coalescence}

The Bayesian parameter updating results are presented in Fig. 7. As can be seen, the parameter distributions have changed. The mean value of uncertain parameters in Table 2. are used to establish the second failure life model. And the mean value of $\mathrm{C}, \mathrm{m}, \mathrm{n}$ are $3.33 \mathrm{E}-10,-0.04588,1.828$, respectively.
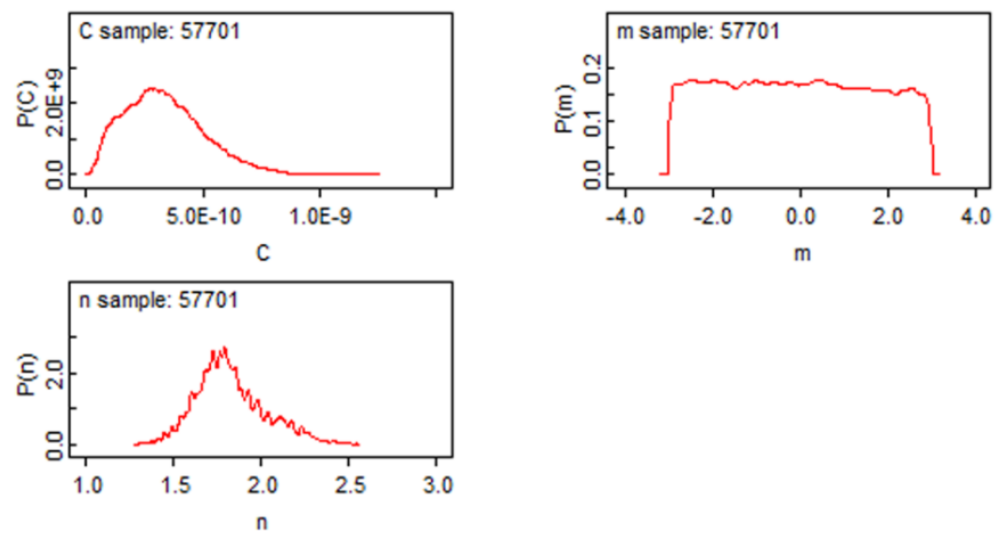

Figure 7. The posteir distribution of uncertain parameters after coalescence

Table 2. The updating results of uncertain parameters after coalescence

\begin{tabular}{|l|l|l|l|l|l|l|}
\hline & Mean & sd & MC_error & Val2.5pc & median & Val97.5pc \\
\hline$C$ & $3.33 \mathrm{E}-10$ & $1.954 \mathrm{E}-10$ & $7.136 \mathrm{E}-12$ & $7.381 \mathrm{E}-10$ & $3.16 \mathrm{E}-10$ & $7.071 \mathrm{E}-10$ \\
\hline$m$ & -0.04588 & 1.716 & 0.03485 & -2.85 & -0.06547 & 2.839 \\
\hline$n$ & 1.828 & 0.1909 & 0.009572 & 1.495 & 1.801 & 2.251 \\
\hline
\end{tabular}


So the mean model for cracks after coalescence can be written as:

$$
\frac{d a}{d N}=3.33 E-10 \Delta K^{-0.04588}(l r)^{1.828}
$$

\subsection{The probability failure model}

According to the PoF model, Monte Carlo simulation of the 10000 sets of parameters has been done to get the probability density distribution histogram of failure life. And the life distribution of probability density curve can be acquired by connecting each midpoint(Fig. 8). The mean failure life are 6223.1 and 7531.82 cycles for cracks considering or ignoring the interactions, respectively. It's apparently that the failure life considering the cracks interactions is shorter than that of ignoring the interactions. For safer failure life assessment of engineering structural parts, the interactions between adjucent cracks are not negligible.

Fig. 9 shows the failure probability curve for cracks considering and ignoring the interactions between adjucent cracks. At first, the two curve have nearly the same failure probabilites before point A where the interaction begins. Then the crack propagation accelerates slowly untile point $\mathrm{B}$. And in the final stage, the cracks propagation further speed up.

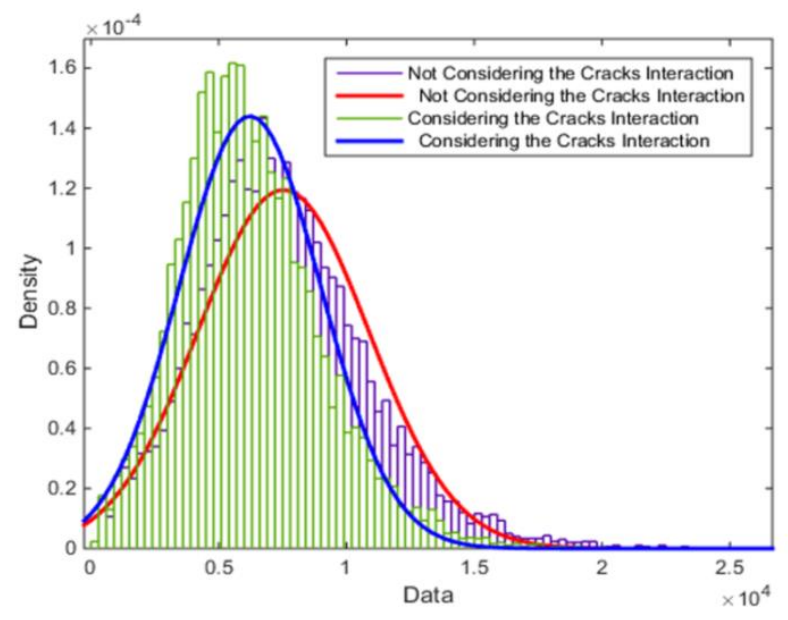

Figure 8. The probability density distributions of cracks

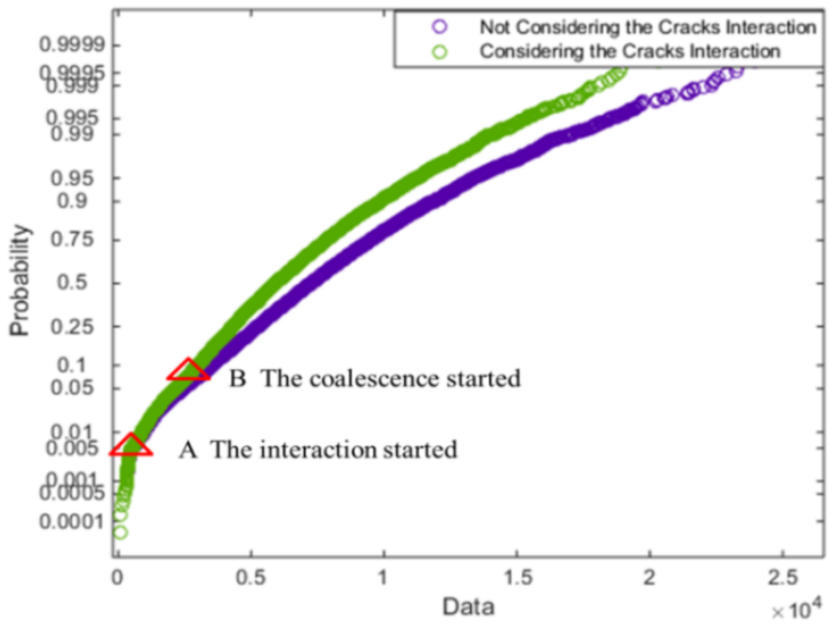

Figure 9. The failure probability curve 


\section{Conclusion}

By investigating the process of interatction and coalescence of adjacent cracks, we found out that the stress intensity factor will increase suddenly in the ligament failure. The time when the coalescence happen was detected through the simulation of the SIF. The posterior distributions of the uncertain parameters were obtained via Bayesian update method. And the MCMC-Gibbs sampling process was realized via WINBUGS.

The PPoF models before and after coalescence were established by Monte Carlo simulation method. Starting from the root cause of failure and taking uncertainty like materials propertie, loading rate and so on into account, the PPOF model can describe the failure life more detail. The probabilistic modeling method can also be applied to other aspect of physic of failure which can greatly reduces the historical data or experimental data needed for the reliability evaluation of small sample products, effectively saving time and cost. The research of coplanar semiellipse cracks propagation in this paper promoted a new thought of studying the interaction and failure propagation in a complex engineering systerm. What's more, the effective life predictting method for multiple fatigue cracks will be a guarantee for personnel and products safety.

\section{References}

1. C.J. Bayley and R. Bell, Int. J. Fatigue, 21, 33-37 (1999)

2. D.L. Su, Mechanical properties of engineering materials (Beijing, China, 2015)

3. J.B. Duan, Chin. Quart. Mech., 32, 6-11 (2011)

4. B.S. Institution, Guidance on methods for assessing the acceptability of flaws in fusion welded structures (London, British, 1991)

5. T.H. Leek and I.C. Howard, Int. J. Pres. Ves. Pip., 68, 181-201 (1996)

6. K. Iida, I.C.F. International Symposium on Fracture Mechanics (Beijing, China, 1983)

7. T. Howes, Russ J Genet, 39, 382-385 (1990)

8. T.H. Leek and I.C. Howard, Int. J. Pres. Ves. Pip., 60, 307-321 (1994)

9. J.R. Black, Phys. Electromig., 1, 142-149 (1974)

10. N.C. Wilmington, I.C. Chen, S. Holland and C. Hu, $23^{\text {rd }}$ Annual Proceedings of Reliability Physics[C], 24-31 (1985)

11. J.F. Archard, J. Appli. Physi, 24, 981-988 (1953)

12. Z. Matik and V. Sruk, The Physics-of-Failure approach in reliability engineering[C], 745-750(2008)

13. M Chookah M. Nuhi and M. Modarres, Relia. Eng. Sys. Saft., 96, 1601-1610 (2011)

14. T. Nagy and T. Turanyi, Relia. Eng. Sys. Saf., 107, 29-34 (2012)

15. E.A. Debartolo and B.M. Hillberry, Int. J. Fatigue, 20, 727-735 (1998)

16. E.J. Moukawsher, M.B. Einimann and A.F. Randt, J. Aircraft, 33, 1003-1013 (2012)

17. T. Swift, Int. J. Fatigue, 16, 75-94 (1994)

18. D.Y. Jeong and J.C. Brewer, Eng. Fract. Mech., 51, 233-238 (1995)

19. J. R. Rice, N. Levy, J. Appl. Mech., 39, 185-194(1972)

20. F. Delale and F. Erdogan, Int. J. Eng. Sci., 19, 1331-1340 (1981)

21. J.H. Yuan, G.J. Tang and X.C. Song, Chin. Quart. Mecha., 21, 8-14 (2000)

22. P. Paris and F. Erdogan, J. Basi. Eng. Trans. ASME, 85, 528-533(1963)

23. M.L. Duan, C.T. Liu and H.J. Zhao, Acta mech. Sinica, 35, 5 (2003)

24. K. Krausz and A.S. Krausz, Int. J. Fracture, 36, 23-28 (1988) 